\title{
Hydranencephaly treatments: retrospective case series and review of the literature
}

\author{
Grace M. Thiong'O, MD, 1,2 Susan S. Ferson, PNP,1 and A. Leland Albright, MD1 \\ 'Division of Neurosurgery, Department of Surgery, Kijabe Hospital, Kijabe, Kenya; and 2University of Toronto, CIGITI, Hospital for \\ Sick Children, Toronto, Ontario, Canada
}

\begin{abstract}
OBJECTIVE The objective of this study was to review treatment options for infants with hydranencephaly and to consider the pros and cons of each treatment modality.

METHODS This paper is a review of hydranencephaly as well as a retrospective analysis evaluating the outcomes of 52 infants with hydranencephaly who were treated at the Kijabe Hospital, Kijabe, Kenya, in one of four ways: ventriculoperitoneal shunt (VPS) insertion, endoscopic choroid plexus coagulation (CPC), open choroid plexectomy (CPIx), and palliative care. The primary outcome measure was control of head size, with the aim of improving patient care. One-year mortality was a secondary outcome.
\end{abstract}

RESULTS Of the 52 patients analyzed, 11 underwent VPS insertion, $17 \mathrm{CPC}, 14 \mathrm{CPIx}$, and 10 were treated palliatively. Head size was controlled at the 3-month evaluation interval in 5 of 7 infants treated with VPS, 10 of 16 of those treated with CPC, 6 of 9 of those treated with CPIx, and 1 of 4 treated palliatively. The number of infants in each category with complete follow-up data that were needed to analyze change in head size was lower than the total number of patients included in each category. Mortality at 1 year of age was 9 of 11 in the VPS group, 14 of 17 in the CPC group, 6 of 14 in the CPIx group, and 7 of 10 in the palliative group.

CONCLUSIONS Head size decreased by $1 \mathrm{~cm}$ or more in similar proportions (62\%-71\%) of infants with hydranencephaly who were treated by VPS insertion, CPC, and CPIx, and progressed in those who received palliative care. Mortality at 1 year of age was similar in infants treated by a VPS, CPC, and palliative care (70\%-82\%), but lower (43\%) in those treated with CPIx.

https://thejns.org/doi/abs/10.3171/2020.3.PEDS19596

KEYWORDS hydranencephaly; VP shunts; choroid plexus coagulation; choroid plexectomy; congenital

$\mathrm{H}$ YDRANENCEPHALY is characterized by the absence or near-total absence of cerebral hemispheres. It is thought to result from destruction of previously developing brain secondary to in-utero bilateral vascular occlusion, although intrauterine infections, maternal exposure to drugs, and genetic factors may play a role. ${ }^{1,2}$ The incidence of hydranencephaly in developed countries is less than 1 in 10,$000 ;{ }^{3}$ its rate in developing countries is unknown. Nevertheless, the largest reported series prior to this paper was 15 patients treated in Malawi. ${ }^{4}$ Patients diagnosed with hydranencephaly are usually blind, deaf, and suffer marked developmental delays and intellectual deficits, spastic quadriparesis, and progressive macrocephaly. ${ }^{1}$

The life expectancy of infants with hydranencephaly is short, with 2-year mortality estimated at $84 \% .^{1}$ Death results from a myriad of complications, including aspiration pneumonia, malnutrition, and infections. Morbidity is compounded by scalp erosion resulting from difficulty in caring for infants with grossly enlarged heads.

Hydranencephaly frequently requires treatment because of the raised intracranial pressure and macrocephaly that progress to extreme degrees and severely hamper child care. Treatment of these infants involves an acknowledgment that the "best" treatment is unknown and consideration of the ethical issues as to whether treatment should be given because of the poor neurological prognosis and high mortality rate. In rural Kenya, another factor to be considered is the delay of treatment caused by difficulties in accessing specialized healthcare. ${ }^{5}$ This paper will compare 4 treatments of hydranencephaly and their outcomes.

ABBREVIATIONS $\mathrm{CPC}=$ choroid plexus coagulation; $\mathrm{CPIx}=$ choroid plexectomy; OFC = occipitofrontal circumference; VPS = ventriculoperitoneal shunt. SUBMITTED October 8, 2019. ACCEPTED March 20, 2020.

INCLUDE WHEN CITING Published online May 15, 2020; DOI: 10.3171/2020.3.PEDS19596. 


\section{Methods}

\section{Literature Review}

A search on PubMed was conducted using the MeSH terms "hydranencephaly" and "treatment", as well as a separate search on the Ovid database using the $\mathrm{MeSH}$ subheadings "hydranencephaly" and "treat*" Inclusion criteria consisted of neurosurgical papers describing treatment of hydranencephaly. From the articles that discussed multiple subheadings, e.g., hydranencephaly plus extreme hydrocephalus, only data on hydranencephaly were extracted. Studies that did not make a distinction between hydranencephaly, near-hydranencephaly, and extreme or maximal hydrocephalus were excluded. All the data are from pediatric patients. Nonneurosurgical papers that focused on obstetric or in utero treatment options offered for hydranencephaly, hemihydranencephaly, and extreme hydrocephalus, and foreign language papers were excluded from this review. Duplicate references were deleted. Cross-referencing identified an additional number of articles.

\section{Single-Institution Study (Kijabe Hospital Kenya)}

A retrospective, single-center study was performed at the Kijabe Hospital in Kijabe, Kenya. In-hospital data were collected prospectively in a study approved by the Kijabe Hospital IRB. The study included all patients treated with hydranencephaly between the years 2010 and 2014. Patients were separated into 4 groups according to the initial treatment modality offered: ventriculoperitoneal shunt (VPS) insertion, endoscopic choroid plexus coagulation (CPC), open choroid plexectomy (CPlx), and palliative care.

Patient data were obtained from in-hospital and mobile clinic follow-up charts and calls to patients' family mobile phones. Data obtained included head circumference 3 months after intervention and mortality at 1 year of age.

Selection criteria for each treatment category were individualized for every child based on factors such as presence of scalp wounds along the projected shunt trajectory necessitating exclusion from VPS insertion, patient proximity to the hospital (as this would determine likelihood of returning to the center for follow-up), and family input after discussing the goals of care. Palliation was encouraged in patients found to be unfit for surgery, such as in severely undernourished infants with concomitant comorbidity.

The goals-of-care discussion with the family included the fact that the aim of surgery was not necessarily to prolong life and would mainly help with head size control and consequently ease patient care.

The occipitofrontal circumference (OFC) was measured by 3 registered nurses dedicated to working in the neurosurgery unit, and led by an author on the paper (S.S.F.).

After the 3-month review, the frequency of clinic follow-up decreased, but all families remained accessible by mobile phone coverage in their home or via a close relative. Phone calls to the mobile phones were made to obtain a response to the mortality question.

The CPC technique at Kijabe has previously been de- scribed ${ }^{6}$ Our CPlx technique involved performing a quadrangular craniotomy at the right coronal suture, a quadrangular dural opening based at the midline, aspiration of CSF down to the level of the thalami, coagulation and excision of all visible choroid plexus from both hemispheres under microscopic magnification, and then replacement of CSF with warm Ringer's lactate solution and closure of the dura and scalp.

Fisher's exact test for nonparametric statistical analysis was performed in $\mathrm{R}$ software to determine the associations among the 4 treatment categories.

\section{Results \\ Literature Search}

The results of the literature search are presented in Fig. 1 in accordance with PRISMA guidelines. ${ }^{7}$ A total of 7 articles ${ }^{1,4,6,8-11}$ were identified for review in this paper. From these studies, 20 of 36 children $(55.5 \%)$ were offered endoscopic CPC as the initial treatment. Only 1 of 36 patients in the previous literature were initially treated using the CPlx technique. Twenty-two (61.1\%) of the total 36 cases were surgically managed in Africa.

\section{Case Series}

At Kijabe Hospital, a total of 52 patients were studied. VPS insertion was performed in 11 infants, endoscopic CPC in 17, open CPlx in 14, and palliative care was given to 10 .

OFCs before treatment were similar in the 4 groups. Pretreatment range of head circumferences and mean circumferences were as follows: VPS group 43-64 cm, mean $52 \mathrm{~cm}$; CPC group 43-61 cm, mean $52 \mathrm{~cm}$; CPlx group $40-61 \mathrm{~cm}$, mean $51 \mathrm{~cm}$; and palliative group 41-66 cm, mean $49 \mathrm{~cm}$. There was no evident difference in the proportion of patients with controlled head size at the 3-month evaluation by any surgical procedure: $5 / 7$ infants treated with a VPS, 10/16 of those treated with CPC, and 6/9 of those treated with CPlx. Head size continued to enlarge in 3 of the 4 infants treated palliatively (Table 1). Fisher's exact test yielded a $\mathrm{p}$ value of 0.5 for the comparison across all 4 treatments.

The mortality rate at 1-year was identical $(82 \%)$ in both the VPS and CPC groups, and was 70\% (7/10) in the palliative care group, but substantially lower $(43 \%, 8 / 14)$ in the CPlx group (Table 2). All deaths occurred at home; their causes were unknown.

\section{Discussion}

CPC and VPS insertion are the most common treatment modalities reported in the literature for the treatment of hydranencephaly. $\mathrm{CPlx}$ remains a seldom-used technique despite a fairly recent randomized trial concluding that CPlx reduces further neurosurgical intervention in children both with hydranencephaly and near hydranencephaly. ${ }^{12}$

Our paper discusses 4 hydranencephaly treatment modalities: VPS insertion, CPIx, CPC, and palliation. VPSs are more available in Kenya and across Africa than are endoscopic procedures. However, shunt infections are frequent in infants with hydranencephaly because of their 


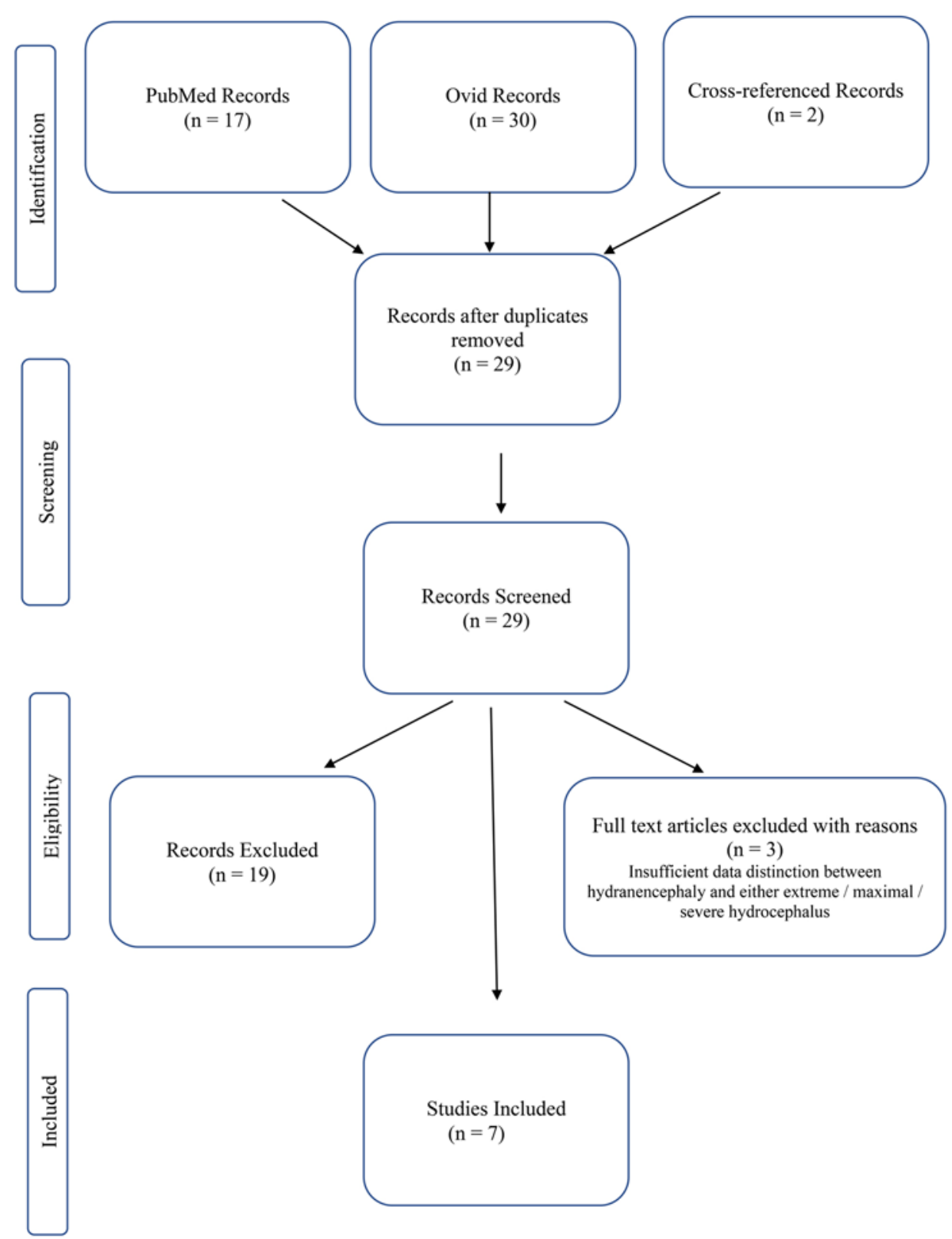

FIG. 1. Flow diagram illustrating the identification process of the literature record review. ${ }^{7}$ Figure is available in color online only.

malnutrition and thin scalps. The long-term function of shunts in infants with hydranencephaly will be difficult to determine because of the high mortality within the first year, among other reasons.

With advances in endoscopic instruments and techniques, CPC has evolved as a second treatment option for children with hydranencephaly. Although endoscopic CPC for hydranencephaly was reported in 1981, with success in 2 of 3 infants, ${ }^{8}$ subsequent cases were not reported until 2010, when Malheiros et al. reported the successful use of CPC to treat hydranencephaly in 8 of 10 infants. ${ }^{12}$

Open CPlx was initially reported by Dandy but the procedure resulted in the deaths of 3 of the 4 infants, a mortality rate that likely led to the abandonment of the procedure for decades thereafter..$^{13}$ In developing countries, one advantage of CPlx over shunts is the avoidance of shunt hardware with its potential for infection, disconnection, and skin erosion.

Palliative care, rather than a surgical procedure, was offered for infants with complex comorbidity such as extreme malnutrition, poor feeding, lethargy, and concomi-

TABLE 1. Head size control at the 3-month follow-up evaluation

\begin{tabular}{lccccc}
\hline \multicolumn{1}{c}{ Patient Variable } & VPS & CPC & CPLx & Palliative \\
\hline No. with controlled head size at 3 mos & 5 & 10 & 6 & 1 \\
\hline Total no. with recorded OFC & 7 & 16 & 9 & 4 \\
\hline No. with controlled head size (\%) & 71 & 62.5 & 67 & 25 \\
\hline
\end{tabular}


TABLE 2. Summary of the results

\begin{tabular}{lrrrc}
\hline \multicolumn{1}{c}{ Patient Variable } & VPS & CPC & CPIx & Palliative \\
\hline Total no. analyzed & 11 & 17 & 14 & 10 \\
\hline Alive at 1 yr & 2 & 3 & 8 & 3 \\
\hline Deaths at 1 yr & 9 & 14 & 6 & 7 \\
\hline$\%$ mortality at 1 yr & 82 & 82 & 43 & 70 \\
\hline
\end{tabular}

tant infections, and for infants whose families were economically destitute. Our data found that this subgroup had progressive macrocephaly, as one would expect. Of the 4 treatment methods reported in this paper, none offers superior head size control, as evidenced by the $\mathrm{p}$ value of 0.5 .

In our opinion, ethical questions about treating children with hydranencephaly are at least as difficult as the questions about which surgical procedure to use; the questions are encountered far more often in low- and middle-income countries than in developed ones, because the incidence of hydranencephaly appears to be so much higher in them.

The clinical conundrum encountered when an infant with hydranencephaly presents for medical attention in a developing or developed country is whether to treat the child. Head size at presentation is usually large, often $50 \mathrm{~cm}$ or more, so that the infant has impaired or absent head control. The mean OFC across all 4 subgroups in our study was between 49 and $52 \mathrm{~cm}$. Because extreme macrocephaly impedes an infant's ability to turn his or her head normally from side to side, scalp ulcers often develop and become infected. If the child is treated surgically, head size is usually successfully decreased, but there are costs, i.e., the monetary costs of the operation and hospitalization, and costs of needing parental caregiving for a longer duration. If the child is not treated surgically, there are no monetary costs and the child's mortality rate at 1 year is similar to that if he or she is treated surgically. Societal norms in rural Kenya hold the firm belief that life must be protected at all costs. In the event that treatment was not offered and the head continued to enlarge, the community would be bewildered, and sometimes the child and mother disowned.

\section{Conclusions}

This paper describes the use and results of 4 potential treatment options for infants with hydranencephaly, including the use of CPlx. Control of head size by any of the treatments did not correlate consistently with prolonged survival. Although 4 treatment options are available, the decisions about treatment of hydranencephaly remain an ethical conundrum.

\section{References}

1. Pavone P, Praticò AD, Vitaliti G, et al. Hydranencephaly: cerebral spinal fluid instead of cerebral mantles. Ital J Pediatr. 2014;40:79.
2. Sherer DM, Anyaegbunam A, Onyeije C. Antepartum fetal intracranial hemorrhage, predisposing factors and prenatal sonography: a review. Am J Perinatol. 1998;15(7):431-441.

3. Kurtz AB, Johnson PT. Diagnosis please. Case 7: Hydranencephaly. Radiology. 1999;210(2):419-422.

4. Adeloye A. Hydranencephaly in Malawian children. East Afr Med J. 2000;77(6):316-318.

5. Mansouri A, Chan V, Njaramba V, et al. Sources of delayed provision of neurosurgical care in a rural Kenyan setting. Surg Neurol Int. 2015;6:32.

6. Shitsama S, Wittayanakorn N, Okechi H, Albright AL. Choroid plexus coagulation in infants with extreme hydrocephalus or hydranencephaly. J Neurosurg Pediatr. 2014;14(1):5557.

7. Moher D, Liberati A, Tetzlaff J, Altman DG. Preferred reporting items for systematic reviews and meta-analyses: the PRISMA statement. PLoS Med. 2009;6(7):e1000097.

8. Albright L. Percutaneous choroid plexus coagulation in hydranencephaly. Childs Brain. 1981;8(2):134-137.

9. Kim SY, Cho JH, Kim KH. Endoscopic coagulation of choroid plexus in hydranencephaly. J Korean Neurosurg Soc. 2014;55(6):375-378.

10. Ogiwara H, Uematsu K, Morota N. Obliteration of the choroid plexus after endoscopic coagulation. J Neurosurg Pediatr. 2014;14(3):230-233.

11. Sandberg DI, Chamiraju P, Zoeller G, et al. Endoscopic choroid plexus coagulation in infants with hydranencephaly or hydrocephalus with a minimal cortical mantle. Pediatr Neurosurg. 2012;48(1):6-12.

12. Malheiros JA, Trivelato FP, Oliveira MM, et al. Endoscopic choroid plexus cauterization versus ventriculoperitoneal shunt for hydranencephaly and near hydranencephaly: a prospective study. Neurosurgery. 2010;66(3):459-464.

13. Dandy WE. Extirpation of the choroid plexus of the lateral ventricles in communicating hydrocephalus. Ann Surg. 1918;68(6):569-579.

\section{Disclosures}

The authors report no conflict of interest concerning the materials or methods used in this study or the findings specified in this paper.

\section{Author Contributions}

Conception and design: Albright. Acquisition of data: Thiong'o, Ferson. Analysis and interpretation of data: Thiong'o, Albright. Drafting the article: Thiong'o. Critically revising the article: all authors. Reviewed submitted version of manuscript: Thiong'o, Albright. Approved the final version of the manuscript on behalf of all authors: Thiong'o. Statistical analysis: Thiong'o. Study supervision: Albright.

\section{Correspondence}

Grace Thiong'o: University of Toronto, CIGITI/Hospital for Sick Children, Toronto, ON, Canada. grace.muthonithiongo@ sickkids.ca. 